\title{
LA APLICACIÓN DE LOS MEDIOS AUDIOVISUALES Y SU INFLUENCIA EN LA COMPETENCIA DE COMPRENSIÓN ORAL Y ESCRITA DEL ÁREA DE COMUNICACIÓN EN LOS ESTUDIANTES DE NIVEL PRIMARIO
}

\author{
Laura ALVARADO JORGE \\ Universidad Nacional Mayor de San Marcos \\ liaJ3@hotmail.com
}

\begin{abstract}
RESUMEN
Los recursos audiovisuales fortalecen el proceso de enseñanza aprendizaje, contribuyen a motivar al alumno, a aclarar conceptos y fijar comportamientos a través de un efectivo proceso. La comunicación, es el medio por medio del cual el hombre puede interrelacionarse y conocer su entorno y comunicarse con otros individuos logrando socializarse utilizando una serie de medios como los auditivos, visuales y audiovisuales, que contribuyen significativamente a logra mejores aprendizajes. La Competencia comprensión oral y escrita aportan al estudio un soporte teórico, metodológico y epistemológico tan necesarias para la formación del ser humano. En la investigación se formuló cuatro hipótesis de las cuales ha quedado confirmada por medio de la contrastación de hipótesis que las dimensiones de Aplicación de los Medio Audiovisuales se relacionan positivamente con Competencia de comprensión oral y escrita del área de comunicación.
\end{abstract}

PALABRAS CLAVE

Medios audiovisuales, oral y comprensión de la competencia escrita.

\section{"THE APPLICATION OF THE MIDDLE AUDIOVISUAL AND ITS INFLUENCE ON THE COMPETITION OF UNDERSTANDING ORAL AND WRITTEN COMMUNICATION AREA STUDENTS OF PRIMARY LEVEL}

\section{ABSTRACT}

Audiovisual resources strengthen the teaching-learning process, help to motivate students, to clarify concepts and establish behaviors through an effective process. Communication is the means by which man can interact and learn about their environment and communicate with other individuals making socialize using a variety of means such as auditory, visual and audiovisual, which contribute significantly to achieve better learning. Competition oral and written comprehension study 
provide a theoretical, methodological and epistemological support as necessary for the formation of human beings. In the research four hypotheses of which has been confirmed through hypothesis testing, that the dimensions of Implementation of the Audiovisual Media Competition are positively related to oral and written communication in the area of understanding.

\section{KEYWORDS}

audio-visual media, oral and understanding of the written compentence.

Recibido: $4 / 8 / 2017$

Aprobado: $30 / 11 / 2017$ 


\section{Objetivos del estudio}

\section{Objetivo General}

eterminar en qué medida la aplicación de los medios audiovisuales influyen en
la competencia de comprensión oral y escrita del área de comunicación en los
alumnos del IV ciclo del Nivel Primaria de la Institución Educativa 3092 Kumamo-
to I, Puente Piedra, Lima 2012 .

\section{Objetivos Específicos}

1. Identificar cómo la aplicación de los medios visuales influyen en la Competencia de comprensión oral y escrita del área de comunicación en los alumnos del IV ciclo del Nivel Primaria de la Institución Educativa 3092 Kumamoto I, Puente Piedra, Lima 2012.

2. Determinar de qué manera la aplicación los medios auditivos influyen en la competencia de comprensión oral y escrita del área de comunicación en los alumnos del IV ciclo del Nivel Primaria de la Institución Educativa 3092 Kumamoto I, Puente Piedra, Lima 2012.

3. Conocer en qué medida la aplicación de los medios audiovisuales influyen en la Competencia de comprensión oral y escrita del área de comunicación en los alumnos del IV ciclo del Nivel Primaria de la Institución Educativa 3092 Kumamoto I, Puente Piedra, Lima 2012.

\section{Diseño del estudio}

Correlacional- Causal

La finalidad del presente estudio es someter a comprobación un modelo explicativo que describe relaciones entre las variables en estudio. Se parte de un modelo explicativo establecido como resultado de una investigación previa (Aguado y cols., 1999), el cual identifica variables críticas (de contexto y de proceso) que se relacionan entre sí.

\section{Técnicas e instrumento de recolección de datos}

Para recoger datos respecto a la variable: medios audiovisuales, se aplicó la técnica un cuestionario a través del instrumento de encuesta y la guía de observación del desarrollo de la clase con medios audiovisuales, suministrado a la muestra de investigación conformada por estudiantes y docentes de la institución educativa 3092 Kumamoto I, Puente Piedra- Lima.

\section{Fuente de información}

La fuente de información en el estudio ha sido primaria y personal, ya que se tuvo acceso inmediato para la recolección de datos en forma directa y personal desde la unidad de análisis.

\section{Instrumento}

Para la recolección de datos se utilizó el cuestionario estructurado estuvo dirigido a los docentes para medir la aplicación de los medios audiovisuales, para la variable dependiente 
se obtuvo a través de la observación de medio audiovisual a través de una lectura para evaluar la comprensión y oral y escrita.

\section{Proceso de contrastación de hipótesis}

\section{Estadística de contraste de hipótesis general}

La contrastación de hipótesis utilizó el modelo estadístico de correlación de Pearson para determinar la relación de la variable independiente medios audiovisuales y la variable dependiente Competencia de comprensión Oral y Escrita del Área de comunicación.

Tabla 1

\begin{tabular}{|c|c|c|c|c|}
\hline & & & $\begin{array}{l}\text { Los medios } \\
\text { audiovisuales }\end{array}$ & $\begin{array}{l}\text { La competencia de } \\
\text { comprensión oral y } \\
\text { escrita del área de } \\
\text { comunicación }\end{array}$ \\
\hline \multirow{6}{*}{$\begin{array}{c}\text { R de } \\
\text { Pearson }\end{array}$} & \multirow{3}{*}{$\begin{array}{l}\text { Los medios } \\
\text { audiovisuales }\end{array}$} & $\begin{array}{l}\text { Coeficiente de } \\
\text { correlación }\end{array}$ & 1,000 &, $615^{* *}$ \\
\hline & & Sig. (bilateral) & . & ,003 \\
\hline & & $\mathrm{N}$ & 36 & 36 \\
\hline & \multirow{3}{*}{$\begin{array}{l}\text { La } \\
\text { competencia } \\
\text { de } \\
\text { comprensión } \\
\text { oral y escrita } \\
\text { del área de } \\
\text { comunicación }\end{array}$} & $\begin{array}{l}\text { Coeficiente de } \\
\text { correlación }\end{array}$ &, $615^{* *}$ & 1,000 \\
\hline & & Sig. (bilateral) & ,003 & \\
\hline & & $\mathrm{N}$ & 36 & 36 \\
\hline
\end{tabular}

\section{Interpretación}

En el cuadro anterior, encontramos una significancia bilateral de 0.03 que es menor al nivel de 0,05 previsto para este análisis. Por lo que podemos afirmar que: Sí existe una influencia significativa entre los medios audiovisuales y la competencia de comprensión oral y escrita del área de comunicación. La correlación es directa, significativa y alcanza un nivel de 0.615 que corresponde a una correlación positiva media.

\section{Estadística de contraste de hipótesis específica 1}

La contrastación de hipótesis utilizó la correlación de Pearson entre la dimensión medios visuales y la variable dependiente. 
Tabla 2

Correlaciones

\begin{tabular}{|c|c|c|c|c|}
\hline & & & $\begin{array}{l}\text { Medios } \\
\text { visuales }\end{array}$ & $\begin{array}{c}\text { La competencia de } \\
\text { comprensión oral y } \\
\text { escrita del área de } \\
\text { comunicación }\end{array}$ \\
\hline \multirow{6}{*}{$\begin{array}{c}\text { R de } \\
\text { Pearson }\end{array}$} & \multirow{3}{*}{ Medios visuales } & $\begin{array}{l}\text { Coeficiente de } \\
\text { correlación }\end{array}$ & 1,000 &, $561^{* *}$ \\
\hline & & Sig. (bilateral) & . & ,000 \\
\hline & & $\mathrm{N}$ & 36 & 36 \\
\hline & \multirow{3}{*}{$\begin{array}{l}\text { La competencia de } \\
\text { comprensión oral y } \\
\text { escrita del area de } \\
\text { comunicación }\end{array}$} & $\begin{array}{l}\text { Coeficiente de } \\
\text { correlación }\end{array}$ &, $561^{* *}$ & 1,000 \\
\hline & & Sig. (bilateral) & ,000 & . \\
\hline & & $\mathrm{N}$ & 36 & 36 \\
\hline
\end{tabular}

** La correlación es significativa al nivel 0,01 (bilateral).

\section{Interpretación}

En el cuadro anterior, encontramos una significancia bilateral de 0.00 que es menor al nivel de 0,05 previsto para este análisis. Por lo que podemos afirmar que: Sí existe una influencia significativa entre los medios visuales y la competencia de comprensión oral y escrita del área de comunicación. La correlación es directa, significativa y alcanza un nivel de 0.561 , que corresponde a un nivel inferencial bueno.

\section{Estadística de contraste de hipótesis específica 2}

La contrastación de hipótesis utilizó la correlación de Pearson entre la dimensión los medios auditivos y la variable dependiente competencia de comprensión oral y escrita del área de comunicación.

\section{Tabla 3}

\section{Correlaciones}

\begin{tabular}{|c|c|c|c|c|}
\hline & & & $\begin{array}{l}\text { Medios } \\
\text { auditivos }\end{array}$ & $\begin{array}{c}\text { La competencia } \\
\text { de comprensión } \\
\text { oral y escrita } \\
\text { del área de } \\
\text { comunicación }\end{array}$ \\
\hline \multirow{6}{*}{$\begin{array}{l}\text { R de } \\
\text { Pearson }\end{array}$} & \multirow{3}{*}{ Medios auditivos } & $\begin{array}{l}\text { Coeficiente de } \\
\text { correlación }\end{array}$ & 1,000 &, $522^{* *}$ \\
\hline & & Sig. (bilateral) & . & ,001 \\
\hline & & $\mathrm{N}$ & 36 & 36 \\
\hline & \multirow{3}{*}{$\begin{array}{l}\text { La competencia de } \\
\text { comprensión oral y } \\
\text { escrita del área de } \\
\text { comunicación }\end{array}$} & $\begin{array}{l}\text { Coeficiente de } \\
\text { correlación }\end{array}$ &, $522^{* *}$ & 1,000 \\
\hline & & Sig. (bilateral) & ,001 & . \\
\hline & & $\mathrm{N}$ & 36 & 36 \\
\hline
\end{tabular}

** La correlación es significativa al nivel 0,01 (bilateral). 


\section{Interpretación}

En el cuadro encontramos una significancia bilateral de 0.01 que es menor al nivel de 0,05 previsto para este análisis. Por lo que podemos afirmar que: Sí existe una influencia significativa entre los medios auditivos y la variable dependiente competencia de comprensión oral y escrita del área de comunicación. La correlación es directa, significativa y alcanza un nivel de 0.522 , que corresponde a un nivel inferencial bueno.

\section{Estadística de contraste de hipótesis especíica 3}

La contrastación de hipótesis utilizó la correlación de Pearson entre los medios audiovisuales y la variable dependiente la competencia de comprensión oral y escrita del área de comunicación.

Tabla 4

\section{Correlaciones}

\begin{tabular}{|c|c|c|c|c|}
\hline & & & $\begin{array}{c}\text { Medios } \\
\text { audiovisuales }\end{array}$ & $\begin{array}{l}\text { La competencia } \\
\text { de comprensión } \\
\text { oral y escrita } \\
\text { del área de } \\
\text { comunicación }\end{array}$ \\
\hline \multirow{6}{*}{$\begin{array}{l}\text { R de } \\
\text { Pearson }\end{array}$} & \multirow{3}{*}{$\begin{array}{l}\text { Medios } \\
\text { audiovisuales }\end{array}$} & $\begin{array}{l}\text { Coeficiente de } \\
\text { correlación }\end{array}$ & 1,000 &, $480^{* *}$ \\
\hline & & Sig. (bilateral) & . & ,003 \\
\hline & & $\mathrm{N}$ & 36 & 36 \\
\hline & \multirow{3}{*}{$\begin{array}{l}\text { La competencia de } \\
\text { comprensión oral y } \\
\text { escrita del área de } \\
\text { comunicación }\end{array}$} & $\begin{array}{l}\text { Coeficiente de } \\
\text { correlación }\end{array}$ & $480^{* *}$ & 1,000 \\
\hline & & Sig. (bilateral) & ,003 & . \\
\hline & & $\mathrm{N}$ & 36 & 36 \\
\hline
\end{tabular}

** La correlación es significativa al nivel 0,01 (bilateral).

\section{Interpretación}

En el cuadro encontramos una significancia bilateral de 0.03 que es menor al nivel de 0,05 previsto para este análisis. Por lo que podemos afirmar que: Sí existe una influencia significativa entre los medios audiovisuales y la variable dependiente competencia de comprensión oral y escrita del área de comunicación. La correlación es directa, significativa y alcanza un nivel de 0.480 , que corresponde a un nivel positiva media.

\section{Discusión}

La presente investigación se enfoca en determinar en qué medida los medios audiovisuales influyen en la competencia de comprensión oral y escrita del área de comunicación en los alumnos del IV ciclo del Nivel Primaria de la Institución Educativa 3092 Kumamoto, Puente Piedra, Lima 2012. A continuación, se analizarán los resultados en base a las teorías desarrolladas en el proceso de la investigación.

De esta manera, se observa que el $43.3 \%$ de encuestados, a veces. Esto concuerda con lo establecido por Moran (2009); También, en la tabla estadística, el $51.7 \%$ de los estudiantes 
manifiesta que a veces según lo que nos comenta Rivadeneira (2008) que los resultados obtenidos durante la investigación le permitieron afirmar que existe relación significativa moderada entre hábitos de estudio y rendimiento académico en el área de Comunicación, de manera general se identifica una mediana presencia de hábitos de estudio siendo los más arraigados la planificación adecuada, ambiente de estudio donde el rendimiento se ubica entre excelente y bueno en mayor porcentaje.

Morán (1999) estudió la Influencia de los Medios Didácticos de Comunicación en la Cultura de los estudiantes de los Centros Educativos de Secundaria de Jesús María" Universidad Privada Inca Garcilaso de la Vega: El auge inusitado de los medios de comunicación y su consecuente penetración ideológica permite un trabajo efectivo en la cultura de los estudiantes que está en formación escolar por lo que se puede comprobar que en un $68 \%$ de una muestra de 146 de los estudiantes de los centros educativos no cuentan con medios didácticos que le permita tener una cultura adecuada hacia la información, mientras que 29 estudiantes que corresponde a un $20 \%$ de la muestra manifiestan que si tienen acceso, mientras que de 18 estudiantes que es el 12\% manifiestan que a veces cuentan con la oportunidad de tener acceso y que han contribuido a fortalecer nuestras costumbres, los principios de moral pública, privada, al tiempo que han presentado el proceso educativo como factor decisivo para la cristalización de una sociedad verdaderamente nacional. La televisión, el cine y la radio deben fortalecer en forma progresiva el amor por la familia, la comunidad, la patria y por aquellos valores que nos identifican. Los medios de comunicación social difunden las costumbres y tradiciones que se manifiestan en la vistosidad, diversidad y contrastes de su música, danzas y bailes y en la variedad y tradición de su cocina que conjuga los más diversos platos y bebidas de la gastronomía de sus diferentes regiones.

En relación a los resultados teóricos, las hipótesis señaladas han demostrado que la aplicación de los medios audiovisuales influyen significativamente con la competencia de comprensión oral y escrita del área de comunicación en los estudiantes del IV ciclo del nivel primaria.

\section{Conclusiones}

Las conclusiones a las que se llegó están en relación con la hipótesis, los objetivos y el marco teórico. Los resultados son los siguientes:

1. En la presente investigación, se contestó la hipótesis de que sí existe una influencia significativa entre los medios audiovisuales y la competencia de comprensión oral y escrita del área de comunicación porque se encuentra una significancia bilateral de 0.03 que es menor al nivel de 0,05 para el análisis. Por lo que podemos afirmar que, la correlación es directa, significativa y alcanza un nivel de 0.596 que corresponde a una correlación positiva media.

2. La investigación demuestra que sí existe una influencia significativa entre los medios visuales y la competencia de comprensión oral y escrita del área de comunicación, se encuentra una significancia bilateral de 0.00 que es menor al nivel de 0,05 para 
el análisis la correlación es directa, significativa y alcanza un nivel de 0.561, que corresponde a un nivel inferencial bueno.

3. De la misma manera, los resultados de la investigación ratificaron la hipótesis de que sí existe una influencia significativa entre los medios auditivos y la variable dependiente competencia de comprensión oral y escrita del área de comunicación. Se encuentra una significancia bilateral de 0.01 que es menor al nivel de 0,05 previsto para este análisis. Por lo que podemos afirmar que: Sí existe la correlación es directa, significativa y alcanza un nivel de 0.522 , que corresponde a un nivel inferencial bueno.

4. Los resultados de la investigación demuestran que sí existe una influencia significativa entre los medios audiovisuales y la variable dependiente competencia de comprensión oral y escrita del área de comunicación. Se encuentra una significancia bilateral de 0.03 que es menor al nivel de 0,05 previsto para este análisis. Por lo que podemos afirmar que: la correlación es directa, significativa y alcanza un nivel de 0.480 , que corresponde a un nivel positiva media.

\section{REFERENCIAS BIBLIOGRÁFICAS}

Aparicio, R. y Agustín García M. (1987). Imagen, vídeo y educación. 1ª Edición México: Fondo de cultura económica.

Cabero, J. (2000). Medios Audiovisuales y nuevas tecnologías para la formación del siglo XXI, Murcia: Diego Marin-Edutec.

De Kofler, B. (2001). Cuestiones relativas de los medios audiovisuales. París: UNESCO, 10-13.

Ferrés, J. (2007). Vídeo y educación, Barcelona: Paidós.

Gallego, D. y A., C. (2009). El ordenador como recurso didáctico, Madrid: UNED.

Garcia, A. (2009). Un acercamiento pragmático a la teoría de la acción comunicativa. en: Lima: Revista Ciencias Humanas. UTP. 6. (19).

Gimeno, J. (2001). El curriculum: una reflexión sobre la práctica. Bogotá: Cincel-Kapelusz. Gutiérrez Espada, L. (1982). Historia de los Medios Audiovisuales. Madrid: Ed. Pirámide.

Méndez Torres, I. (1994). El lenguaje oral y escrito en la comunicación. México: Editorial Limusa.

Ministerio de Educación (2010). Diseño Curricular Nacional Básica. Lima: Editorial Ministerio de Educación.

Moran (2009). Influencia de los Medios Didácticos de Comunicación en la Cultura de los estudiantes de los Centros Educativos de Secundaria de Jesús María. Lima: UIGV

Muñoz, J. F. \& Quintero J. (2001). Como desarrollar competencias investigativas en educación. Lima: Editorial Cooperativa Editorial Magisterio. Colección aula abierta, 83-107.

Ogalde C. I y Bordavid N.E. (2001). Los materiales educativos. Medios y recursos de apoyo a la docencia. México: Edit. Trillas S.A.

Prendes, M.P. y Munuera, F. (2008). Medios y recursos en educación especial, Murcia, ICE de la Universidad de Murcia-Diego Marín. 
Reyes, A. (2008). Técnicas y modelos de calidad en el salón de clases. México: Editorial. Trillas, S. A.

Vasquez Rojas, R. (2009). La Ética de los Medios audiovisuales que atenta contra la libertad de expresión. Lima: UIGV. 